\title{
Sequential Treatment of Afatinib and Osimertinib in Patients with Advanced Non-small Cell Lung Cancer Harboring EGFR Mutations: Results from a Real-world Study in South Korea
}

Taeyun Kim

The Armed Forces Goyang Hospital

Tae Won Jang ( $\sim$ jangtw22@hanmail.net )

Kosin University Gospel Hospital

Chang Min Choi

Asan Medical Center

Mi-Hyun Kim

Pusan National University Hospital

Sung Yong Lee

Korea University Guro Hospital

Cheol-Kyu Park

Chonnam National University Hwasun Hospital

Yoon Soo Chang

Gangnam Severance Hospital

Kye Young Lee

Konkuk University Medical Center

Seung Joon Kim

Seoul St. Mary's Hospital

\section{Sei Hoon Yang}

Wonkwang University Medical Center

Jeong Seon Ryu

Inha University Hospital

Jeong Eun Lee

Chungnam National University Hospital

\section{Shin Yup Lee}

Kyungpook National University Chilgok Hospital

Chan Kwon Park

Yeouido St. Mary's Hospital

Sang Hoon Lee

Severance Hospital

\section{Seung Hun Jang}

Hallym University Sacred Heart Hospital

Seong Hoon Yoon 
Pusan National University Yangsan Hospital

\section{Research Article}

Keywords: NSCLC, afatinib, osimertinib, EGFR, real-world data

Posted Date: February 19th, 2021

DOl: https://doi.org/10.21203/rs.3.rs-215079/v1

License: (c) (i) This work is licensed under a Creative Commons Attribution 4.0 International License. Read Full License 


\section{Abstract}

The optimal sequence for administration of epidermal growth factor receptor (EGFR) tyrosine kinase inhibitors (TKIs) for treating non-small cell lung cancer (NSCLC) is still unclear. This study aimed to evaluate the efficacy of sequential afatinib and osimertinib treatment in patients with NSCLC harboring EGFR mutations. Electronic records of patients with EGFR-mutated NSCLC, who were administered afatinib and osimertinib (group A) or other chemotherapy (group B) between October 2014 and October 2019, across 16 hospitals in South Korea were reviewed. The primary outcome, time on treatment (TOT), secondary outcome, and overall survival (OS) were estimated using the Kaplan-Meier method and log-rank test. Multivariate analyses were performed using the Cox proportional hazards model. Of the 737 patients who received frontline afatinib treatment, 360 with complete records were selected (group A:154, group B: 206). The median TOT was 33.9 months (95\% confidence interval [CI]: 24.5-43.3) in group $A$ and 21.3 months (95\% Cl: 19.4-23.1) in group B. The median TOT with afatinib was 12.9 months (95\% Cl: 11.8-14.0) overall, and 15.2 months (95\% Cl: 13.2-17.1) in group A. The 2- and 3-year survival rates were $86.0 \%$ and $69.3 \%$ in group $A$ and $75.9 \%$ and $55.3 \%$ in group B, respectively. Sequential afatinib and osimertinib treatment resulted in better survival rates than treatment with afatinib followed by other chemotherapies. Therefore, this sequential treatment strategy may offer clinical benefits to patients with EGFRmutated NSCLC.

\section{Introduction}

Lung cancer is the leading cause of cancer-related deaths in Korea, accounting for approximately $20 \%$ of all cancerrelated deaths; in 2018, 19,317 people died from the disease ${ }^{1}$. Globally, $18.4 \%$ of all cancer-related deaths were attributable to lung cancer, and approximately 2 million people were newly diagnosed in $2018^{2}$.

Afatinib is a second-generation epidermal growth factor receptor (EGFR) tyrosine kinase inhibitor (TKI) that is suggested as the primary treatment option for progressive, EGFR-mutated NSCLC ${ }^{3}$. A recent head-to-head trial demonstrated that afatinib was superior to gefitinib, a first-generation TKI, showing better progression-free survival (PFS) and time-to-treatment failure (TTF) ${ }^{4}$. Despite having many advantages over standard platinum-based chemotherapy and first-generation EGFR TKIs, mutations that confer resistance to afatinib are important clinical problems. The T790M resistance mutation, which is found in exon 20 of the EGFR gene, has been identified in approximately $50 \%$ of patients receiving afatinib as a first-line therapy ${ }^{5}$. The AURA study showed a high objective response rate (ORR) and encouraging PFS with the third-generation EGFR TKI, osimertinib, in patients with T790Mmutated NSCLC previously treated with another $\mathrm{TKI}^{6}$. Based on studies to date, the expected median period for sequential TKI treatment is approximately 24 months, 13 to 14 months with afatinib and 10 to 13 months with osimertinib $4,6,7$.

Real-world data (RWD) are generally used to monitor post-market safety and adverse events. RWD additionally allow clinical decision-making on patient groups that have been excluded by the generally strict inclusion/exclusion criteria of randomized clinical trials (RCTs). Such patients include elderly patients, those with poor performance status, those harboring uncommon EGFR mutations (i.e., neither the exon 19 deletion [Del19] nor L858R), and those with brain metastases. Thus, RWD may better reflect actual clinical conditions. The GioTag study, a retrospective, observational, and global multicenter study of NSCLC patients who received sequential treatment with afatinib and osimertinib, revealed a median TKI-therapy period of 27.6 months and found that patients with the exon 19 mutation were appropriate candidates for these TKI therapies ${ }^{8}$. In a subgroup analysis of 50 Asian patients, the 
median time on TKI therapy was 46.7 months. Other RWD from South Korea indicated that the median PFS was 19.1 months for afatinib, 13.7 months for gefitinib, and 14.0 months for erlotinib, respectively ${ }^{9}$.

Several studies have been conducted in Asian populations on the cumulative advantage of frontline treatment with afatinib in a real-world setting in NSCLC patients harboring EGFR mutations ${ }^{9-13}$. However, there is still a paucity of RWD regarding the clinical characteristics and outcomes of patients who were treated sequentially with afatinib and osimertinib. Moreover, considering the controversy on whether osimertinib should be used as first-line TKI therapy or second-line TKI therapy after failure of first-or second-generation TKIs, the acquisition of data comparing first-line afatinib followed by second-line osimertinib with other second-line treatments is warranted. In this context, the present study evaluated the clinical characteristics and treatment outcomes in patients with EGFR-mutated NSCLC who received first-line treatment with afatinib and second-line therapy with either osimertinib or other regimens by analyzing RWD in South Korea.

\section{Results}

\subsection{Patient characteristics}

A total of 737 patients with EGFR-mutated advanced-stage NSCLC were enrolled in the study. Of these, we excluded 367 patients: 164 patients continued afatinib therapy, 110 experienced progressive disease with no available data on second-line treatment, 10 refused treatment, 15 discontinued treatment due to afatinib toxicity, and 68 were transferred or lost to follow-up. A final set of 360 eligible patients (154 in group A and 206 in group B) were selected. The study flow chart is depicted in Supplementary Fig. 1. The baseline characteristics of the study participants are summarized in Table 1. The tumor stage was more advanced in group A patients than in group B patients. The latter group experienced a higher percentage of newly appeared or aggravated brain metastases. Other variables were not statistically different between groups $A$ and $B$. At the start of second-line treatments, the presence and type of brain metastasis were well balanced. 
Table 1

Characteristics of study participants

\begin{tabular}{|c|c|c|c|c|}
\hline & \multirow{3}{*}{$\begin{array}{l}\text { First-line treatment } \\
\text { Afatinib } \\
(n=360)\end{array}$} & \multicolumn{3}{|c|}{ Second-line treatment } \\
\hline & & Group A & Group B & $P$ \\
\hline & & $(n=154)$ & $(n=206)$ & \\
\hline Male & $191(53.1 \%)$ & $81(52.6 \%)$ & $110(53.4 \%)$ & 0.880 \\
\hline Age & & & & 0.441 \\
\hline$<65$ & $202(56.1 \%)$ & $90(58.4 \%)$ & $112(54.4 \%)$ & \\
\hline$\geq 65$ & $158(43.9 \%)$ & $64(41.6 \%)$ & $94(45.6 \%)$ & \\
\hline Stage§ & & & & 0.003 \\
\hline 3 and $4 \mathrm{~A}$ & $211 / 359(58.8 \%)$ & $77(50.0 \%)$ & $134 / 205(65.4 \%)$ & \\
\hline $4 \mathrm{~B}$ & $148 / 359(41.2 \%)$ & $77(50.0 \%)$ & $71 / 205(34.6 \%)$ & \\
\hline Smoking & & & & 0.973 \\
\hline Never & $225 / 357(63.0 \%)$ & $96(62.3 \%)$ & $129 / 203(63.5 \%)$ & \\
\hline Former & $98 / 357(27.5 \%)$ & $43(27.9 \%)$ & $55 / 203(27.1 \%)$ & \\
\hline Current & $34 / 357(9.5 \%)$ & $15(9.7 \%)$ & $19 / 203(9.4 \%)$ & \\
\hline \multicolumn{5}{|l|}{ ECOG PS } \\
\hline 0 and 1 & $305 / 328(93.0 \%)$ & $125 / 130(96.2 \%)$ & $180 / 198(90.9 \%)$ & 0.079 \\
\hline$\geq 2$ & $23 / 328(7.0 \%)$ & $5 / 130(3.8 \%)$ & 18/198 (9.1\%) & \\
\hline \multicolumn{5}{|l|}{ Tissue type } \\
\hline Adenocarcinoma & $352(97.8 \%)$ & $150(97.4 \%)$ & $202(98.1 \%)$ & 0.728 \\
\hline Others 9 & $8(2.2 \%)$ & $4(2.6 \%)$ & $4(1.9 \%)$ & \\
\hline Presence of EGFR mutation & $339 / 339(100 \%)$ & $145 / 145(100 \%)$ & $194 / 194(100 \%)$ & - \\
\hline EGFR mutation & & & & 0.149 \\
\hline
\end{tabular}

Data are presented as numbers (percentages), unless otherwise stated.

Patients in group A received sequential treatment with afatinib and osimertinib, while patients in group $B$ received other therapies following first-line afatinib treatment.

§ Tumor stage was classified based on 8th edition of the American Joint Committee on Cancer staging system.

9 Other tissue types included squamous cell carcinoma in 2 patients, adenosquamous cell carcinoma in 2 patients, and non-small cell lung cancer in 4 patients.

† Patients not presenting with EGFR Del19 and L858R mutations, including de novo T790M mutation, are classified as the "Others" group.

ECOG PS, Eastern Cooperative Oncology Group performance status; EGFR, epidermal growth factor receptor; Del19, deletion 19; meta., metastasis; adj., adjustment 


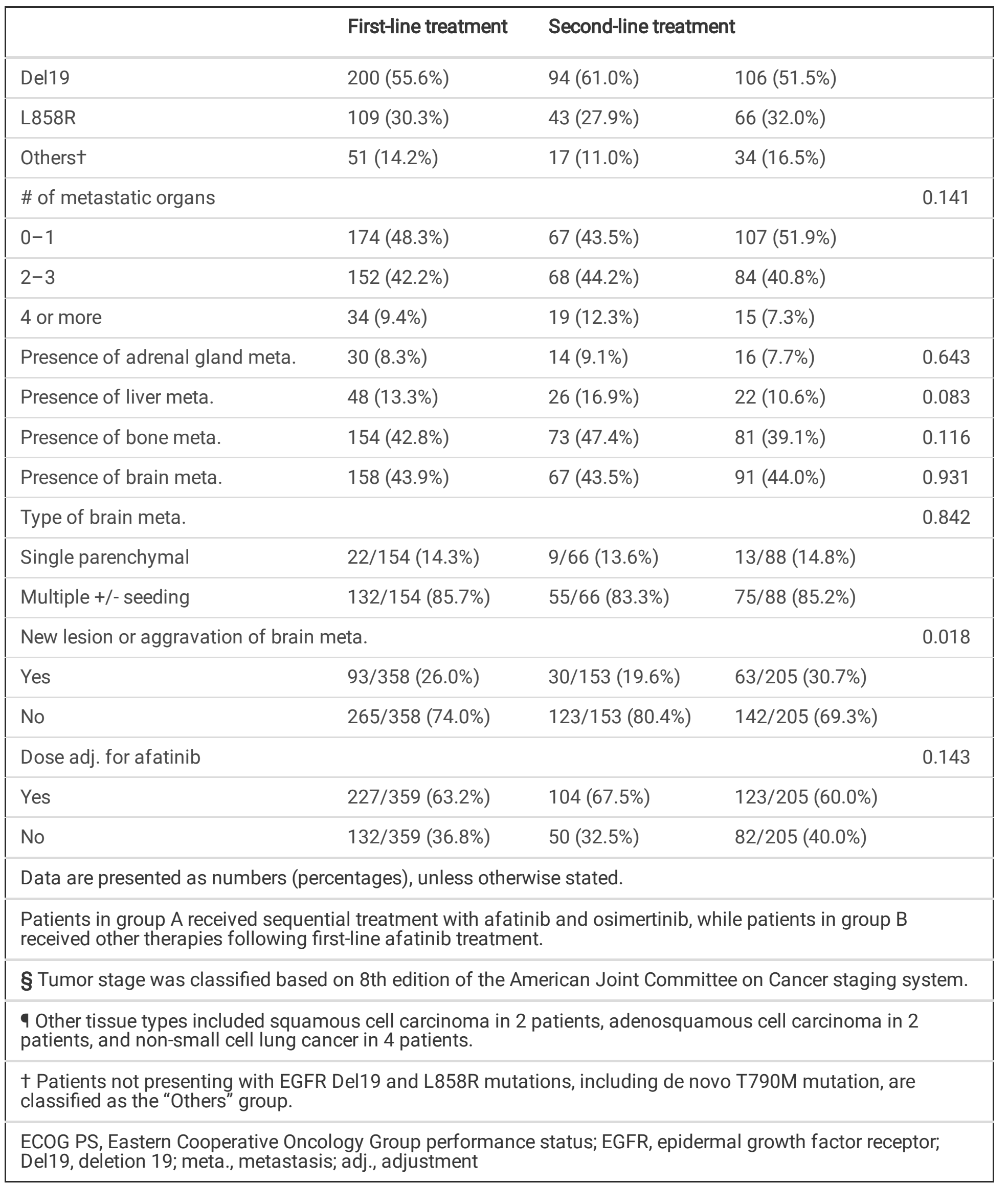

\subsection{Median TOT during first- and second-line treatment}

The results of median TOT during the first- and second-line treatments are summarized in Table 2 . The median TOT in all patients was estimated to be 26.4 (95\% Cl: 24.2-30.4) months. The overall TOT in group A was 33.9 (95\% Cl: 
24.5-43.3) months, which was significantly longer than that in group B (21.3 [95\% Cl: 19.4-23.1] months, $P<0.001$, Fig. 1). 
Table 2

Overall median time-on-treatment in patients who received first- and second-line treatments

\begin{tabular}{|c|c|c|c|c|c|c|}
\hline & $\begin{array}{l}\text { Total } \\
(n=360)\end{array}$ & $P$ & $\begin{array}{l}\text { Group A } \\
(n=154)\end{array}$ & $P$ & $\begin{array}{l}\text { Group B } \\
(n=206)\end{array}$ & $P$ \\
\hline Overall & $\begin{array}{l}26.4(24.2- \\
30.4)\end{array}$ & & $\begin{array}{l}33.9(24.5- \\
43.3)\end{array}$ & & $\begin{array}{l}21.3(19.4- \\
23.1)\end{array}$ & \\
\hline Age & & 0.879 & & 0.273 & & 0.522 \\
\hline$<65$ & $\begin{array}{l}26.2(22.5- \\
30.5)\end{array}$ & & $\begin{array}{l}27.0(20.5- \\
34.9)\end{array}$ & & $\begin{array}{l}21.7(17.7- \\
25.7)\end{array}$ & \\
\hline$\geq 65$ & $\begin{array}{l}27.4(21.9- \\
33.7)\end{array}$ & & $\begin{array}{l}41.1(31.2- \\
51.0)\end{array}$ & & $20.3(18,1-22.5)$ & \\
\hline Sex & & 0.226 & & 0.888 & & 0.070 \\
\hline Male & $\begin{array}{l}24.4(21.3- \\
30.4)\end{array}$ & & $\begin{array}{l}33.8(18.5- \\
49.0)\end{array}$ & & $\begin{array}{l}19.9(17.2- \\
22.5)\end{array}$ & \\
\hline Female & $27.6(25.0-33.9)$ & & $\begin{array}{l}35.2(29.5- \\
40.9)\end{array}$ & & $\begin{array}{l}23.5(19.6- \\
27.4)\end{array}$ & \\
\hline ECOG PS & & 0.019 & & 0.744 & & 0.081 \\
\hline 0 or 1 & $\begin{array}{l}27.4(24.9- \\
31.0)\end{array}$ & & $\begin{array}{l}33.9(28.9- \\
38.8)\end{array}$ & & $\begin{array}{l}21.5(18.5- \\
24.4)\end{array}$ & \\
\hline$\geq 2$ & 19.8 (14.2-NA) & & 20.1 (NA-NA) & & $16.7(13.0-20.3)$ & \\
\hline Stage§ & & 0.415 & & 0.141 & & 0.207 \\
\hline 3 and $4 A$ & $\begin{array}{l}27.5(24.7- \\
33.8)\end{array}$ & & $\begin{array}{l}39.5(32.9- \\
46.1)\end{array}$ & & $\begin{array}{l}21.7(18.6- \\
24.8)\end{array}$ & \\
\hline $4 \mathrm{~B}$ & $\begin{array}{l}24.8(21.4- \\
30.4)\end{array}$ & & $\begin{array}{l}27.1(20.1- \\
34.2)\end{array}$ & & $\begin{array}{l}19.8(17.5- \\
22.1)\end{array}$ & \\
\hline Smoking & & 0.547 & & 0.291 & & 0.189 \\
\hline Never & $27.6(25.0-31.2)$ & & $\begin{array}{l}33.9(23.6- \\
44.2)\end{array}$ & & $\begin{array}{l}22.5(18.6- \\
26.4)\end{array}$ & \\
\hline Former & $\begin{array}{l}24.0(20.9- \\
31.0)\end{array}$ & & $\begin{array}{l}27.1(15.7- \\
38.5)\end{array}$ & & $\begin{array}{l}21.3(18.4- \\
24.1)\end{array}$ & \\
\hline
\end{tabular}

Data are presented as months (95\% confidence intervals), unless otherwise stated.

Patients in group A received sequential treatment with afatinib and osimertinib, while patients in group $B$ received other therapies following first-line afatinib treatment.

§ Tumor stage was classified based on 8th edition of the American Joint Committee on Cancer staging system.

9 Other tissue types included squamous cell carcinoma in 2 patients, adenosquamous cell carcinoma in 2 patients, and non-small cell lung cancer in 4 patients.

† Patients not presenting with EGFR Del19 and L858R mutations, including de novo T790M mutation, are classified as the "Others" group.

ECOG PS, Eastern Cooperative Oncology Group performance status; EGFR, epidermal growth factor receptor; Del19, deletion 19; meta., metastasis; adj., adjustment; NA, not-available. 


\begin{tabular}{|c|c|c|c|c|c|c|}
\hline & $\begin{array}{l}\text { Total } \\
(n=360)\end{array}$ & $P$ & $\begin{array}{l}\text { Group A } \\
(n=154)\end{array}$ & $P$ & $\begin{array}{l}\text { Group B } \\
(n=206)\end{array}$ & $P$ \\
\hline Current & 22.2 (16.0-NA) & & $\begin{array}{l}47.0(27.5- \\
66.5)\end{array}$ & & $12.3(8.4-16.2)$ & \\
\hline Tissue type & & 0.027 & & 0.415 & & 0.001 \\
\hline Adenocarcinoma & $\begin{array}{l}26.5(24.4- \\
30.8)\end{array}$ & & $\begin{array}{l}35.2(25.7- \\
44.6)\end{array}$ & & $21.4(19.0-23.7)$ & \\
\hline Others 9 & 16.6 (12.2-NA) & & 27.1 (NA-NA) & & $8.9(2.4-15.4)$ & \\
\hline EGFR mutation & & 0.791 & & 0.855 & & 0.730 \\
\hline Del19 & $\begin{array}{l}26.9(24.1- \\
29.8)\end{array}$ & & $\begin{array}{l}35.2(24.3- \\
46.0)\end{array}$ & & $\begin{array}{l}21.4(18.7- \\
24.1)\end{array}$ & \\
\hline L858R & $\begin{array}{l}26.4(22.8- \\
30.1)\end{array}$ & & $\begin{array}{l}27.6(19.2- \\
36.1)\end{array}$ & & $\begin{array}{l}22.5(12.8- \\
32.2)\end{array}$ & \\
\hline Otherst & $\begin{array}{l}23.5(15.1- \\
31.8)\end{array}$ & & $\begin{array}{l}42.1(28.9- \\
55.3)\end{array}$ & & $\begin{array}{l}18.4(15.5- \\
21.3)\end{array}$ & \\
\hline \# of metastatic organs & & 0.001 & & 0.009 & & 0.002 \\
\hline $0-1$ & $\begin{array}{l}31.0(25.6- \\
36.4)\end{array}$ & & $\begin{array}{l}42.1 \text { (34.3- } \\
49.9)\end{array}$ & & $\begin{array}{l}24.9(19.5- \\
30.4)\end{array}$ & \\
\hline $2-3$ & $\begin{array}{l}23.5(19.2- \\
27.8)\end{array}$ & & $\begin{array}{l}31.0(23.6- \\
38.5)\end{array}$ & & $\begin{array}{l}19.4(16.7- \\
22.0)\end{array}$ & \\
\hline 4 or more & $19.8(15.0-24.5)$ & & $\begin{array}{l}22.0(14.6- \\
29.4)\end{array}$ & & $\begin{array}{l}16.2(12.3- \\
20.1)\end{array}$ & \\
\hline Adrenal gland meta. & & 0.300 & & 0.332 & & 0.389 \\
\hline Yes & 19.4 (14.9-NA) & & $32.8(9.6-55.9)$ & & $16.2(13.0-19.4)$ & \\
\hline No & $\begin{array}{l}26.5(24.4- \\
30.5)\end{array}$ & & $\begin{array}{l}33.9(23.9- \\
43.8)\end{array}$ & & $\begin{array}{l}21.4(19.1- \\
23.7)\end{array}$ & \\
\hline Liver meta. & & $<.001$ & & $<.001$ & & 0.001 \\
\hline
\end{tabular}

Data are presented as months (95\% confidence intervals), unless otherwise stated.

Patients in group A received sequential treatment with afatinib and osimertinib, while patients in group B received other therapies following first-line afatinib treatment.

§ Tumor stage was classified based on 8th edition of the American Joint Committee on Cancer staging system.

9 Other tissue types included squamous cell carcinoma in 2 patients, adenosquamous cell carcinoma in 2 patients, and non-small cell lung cancer in 4 patients.

† Patients not presenting with EGFR Del19 and L858R mutations, including de novo T790M mutation, are classified as the "Others" group.

ECOG PS, Eastern Cooperative Oncology Group performance status; EGFR, epidermal growth factor receptor; Del19, deletion 19; meta., metastasis; adj., adjustment; NA, not-available. 


\begin{tabular}{|c|c|c|c|c|c|c|}
\hline & $\begin{array}{l}\text { Total } \\
(n=360)\end{array}$ & $P$ & $\begin{array}{l}\text { Group A } \\
(n=154)\end{array}$ & $P$ & $\begin{array}{l}\text { Group B } \\
(n=206)\end{array}$ & $P$ \\
\hline Yes & $\begin{array}{l}19.1(15.5- \\
24.4)\end{array}$ & & $\begin{array}{l}21.4(18.1- \\
24.7)\end{array}$ & & $13.8(8.6-19.0)$ & \\
\hline No & $27.6(25.0-33.7)$ & & $\begin{array}{l}41.1(34.3- \\
47.9)\end{array}$ & & $\begin{array}{l}21.5(19.1- \\
23.9)\end{array}$ & \\
\hline Bone meta. & & 0.005 & & 0.023 & & 0.004 \\
\hline Yes & $\begin{array}{l}21.4(20.1- \\
26.4)\end{array}$ & & $\begin{array}{l}27.6(21.4- \\
33.8)\end{array}$ & & $\begin{array}{l}19.4(17.2- \\
21.5)\end{array}$ & \\
\hline No & $\begin{array}{l}30.5(26.5- \\
34.5)\end{array}$ & & $\begin{array}{l}42.1(33.6- \\
50.5)\end{array}$ & & $\begin{array}{l}23.5(18.5- \\
28.5)\end{array}$ & \\
\hline Brain meta. & & 0.280 & & 0.343 & & 0.630 \\
\hline Yes & $\begin{array}{l}24.4(21.4- \\
29.0)\end{array}$ & & $\begin{array}{l}31.0(22.6- \\
39.5)\end{array}$ & & $19.9(18.0-21.8)$ & \\
\hline No & $27.6(25.0-33.8)$ & & $\begin{array}{l}35.4(27.4- \\
43.4)\end{array}$ & & $\begin{array}{l}22.5(19.1- \\
25.9)\end{array}$ & \\
\hline Type of brain meta. & & 0.463 & & 0.978 & & 0.265 \\
\hline Single parenchymal & $25.3(8.5-42.1)$ & & 42.4 (NA-NA) & & $\begin{array}{l}21.4(19.9- \\
22.9)\end{array}$ & \\
\hline Multiple +/- seeding & $\begin{array}{l}24.4(21.1- \\
27.7)\end{array}$ & & $\begin{array}{l}31.0(21.5- \\
40.6)\end{array}$ & & $\begin{array}{l}19.4(16.3- \\
22.4)\end{array}$ & \\
\hline $\begin{array}{l}\text { New lesion or } \\
\text { aggravation } \\
\text { of brain meta. }\end{array}$ & & 0.007 & & 0.362 & & 0.069 \\
\hline Yes & $\begin{array}{l}21.4(19.1- \\
23.6)\end{array}$ & & $\begin{array}{l}27.7(17.2- \\
38.2)\end{array}$ & & $\begin{array}{l}18.9(14.9- \\
22.9)\end{array}$ & \\
\hline No & $\begin{array}{l}27.6(23.8- \\
31.4)\end{array}$ & & $\begin{array}{l}35.2(27.3- \\
43.0)\end{array}$ & & $\begin{array}{l}22.5(18.5- \\
26.5)\end{array}$ & \\
\hline Dose adj. for afatinib & & 0.016 & & 0.351 & & 0.025 \\
\hline
\end{tabular}

Data are presented as months ( $95 \%$ confidence intervals), unless otherwise stated.

Patients in group A received sequential treatment with afatinib and osimertinib, while patients in group B received other therapies following first-line afatinib treatment.

§ Tumor stage was classified based on 8th edition of the American Joint Committee on Cancer staging system.

9 Other tissue types included squamous cell carcinoma in 2 patients, adenosquamous cell carcinoma in 2 patients, and non-small cell lung cancer in 4 patients.

† Patients not presenting with EGFR Del19 and L858R mutations, including de novo T790M mutation, are classified as the "Others" group.

ECOG PS, Eastern Cooperative Oncology Group performance status; EGFR, epidermal growth factor receptor; Del19, deletion 19; meta., metastasis; adj., adjustment; NA, not-available. 


\begin{tabular}{|c|c|c|c|c|c|c|}
\hline & $\begin{array}{l}\text { Total } \\
(n=360)\end{array}$ & $P$ & $\begin{array}{l}\text { Group A } \\
(n=154)\end{array}$ & $P$ & $\begin{array}{l}\text { Group B } \\
(n=206)\end{array}$ & $P$ \\
\hline Yes & $\begin{array}{l}27.6(23.4- \\
31.8)\end{array}$ & & $\begin{array}{l}35.2(23.9- \\
46.4)\end{array}$ & & $\begin{array}{l}22.5(19.4- \\
25.6)\end{array}$ & \\
\hline No & $24.9(20.0-30.1)$ & & $\begin{array}{l}32.8(24.2- \\
41.3)\end{array}$ & & $\begin{array}{l}18.1(15.3- \\
20.8)\end{array}$ & \\
\hline \multicolumn{7}{|c|}{ Data are presented as months ( $95 \%$ confidence intervals), unless otherwise stated. } \\
\hline \multicolumn{7}{|c|}{$\begin{array}{l}\text { Patients in group A received sequential treatment with afatinib and osimertinib, while patients in group B } \\
\text { received other therapies following first-line afatinib treatment. }\end{array}$} \\
\hline \multicolumn{7}{|c|}{ § Tumor stage was classified based on 8th edition of the American Joint Committee on Cancer staging system. } \\
\hline \multicolumn{7}{|c|}{$\begin{array}{l}\text { Other tissue types included squamous cell carcinoma in } 2 \text { patients, adenosquamous cell carcinoma in } 2 \\
\text { patients, and non-small cell lung cancer in } 4 \text { patients. }\end{array}$} \\
\hline \multicolumn{7}{|c|}{$\begin{array}{l}\text { † Patients not presenting with EGFR Del19 and L858R mutations, including de novo T790M mutation, are } \\
\text { classified as the "Others" group. }\end{array}$} \\
\hline \multicolumn{7}{|c|}{$\begin{array}{l}\text { ECOG PS, Eastern Cooperative Oncology Group performance status; EGFR, epidermal growth factor receptor; } \\
\text { Del19, deletion 19; meta., metastasis; adj., adjustment; NA, not-available. }\end{array}$} \\
\hline
\end{tabular}

\subsection{Respective median TOT (TOT-1 and TOT-2) according to first- and second-line treatments}

The individual median TOTs for the first- and second-line treatments are depicted in Table 3. The estimated median TOT-1 was 12.9 (95\% Cl: 11.8-14.0) months in all patients, 15.2 (95\% Cl: 13.2-17.1) months in group A patients, and 11.0 (95\% Cl: 9.3-12.7) months in group B patients. The difference in TOT-1 between groups A and B was statistically significant $(P<0.001$, Fig. 2). The median TOT-2 in groups $A$ and $B$ patients was 11.2 (95\% Cl: 8.9-13.6) and 5.1 (95\% Cl: 4.4-6.6) months, respectively, and this difference was significant $(P<0.001)$. 
Table 3

Respective median time-on-treatment in patients who received first- and second-line treatments

\begin{tabular}{|c|c|c|c|c|c|c|c|c|c|c|}
\hline & \multicolumn{6}{|c|}{ First-line treatment } & \multicolumn{4}{|c|}{ Second-line treatment } \\
\hline & $\begin{array}{l}\text { Total } \\
(n= \\
360)\end{array}$ & $P$ & $\begin{array}{l}\text { Group } \\
A(n= \\
154)\end{array}$ & $P$ & $\begin{array}{l}\text { Group } \\
B(n= \\
206)\end{array}$ & $P$ & $\begin{array}{l}\text { Group } \\
A(n= \\
154)\end{array}$ & $P$ & $\begin{array}{l}\text { Group } \\
\text { B }(n=) \\
206)\end{array}$ & $P$ \\
\hline Overall & $\begin{array}{l}12.9 \\
(11.8- \\
14.0)\end{array}$ & & $\begin{array}{l}15.2 \\
(13.2- \\
17.1)\end{array}$ & & $\begin{array}{l}11.0 \\
(9.3- \\
12.7)\end{array}$ & & $\begin{array}{l}11.2 \\
(8.9- \\
13.6)\end{array}$ & & $\begin{array}{l}5.1 \\
(4.4- \\
6.6)\end{array}$ & \\
\hline Age & & 0.872 & & 0.120 & & 0.060 & & 0.563 & & 0.536 \\
\hline$<65$ & $\begin{array}{l}13.2 \\
(11.8- \\
14.7)\end{array}$ & & $\begin{array}{l}14.9 \\
(12.8- \\
17.0)\end{array}$ & & $\begin{array}{l}11.0 \\
(9.3- \\
12.7)\end{array}$ & & $\begin{array}{l}10.8 \\
(7.8- \\
13.8)\end{array}$ & & $\begin{array}{l}4.4 \\
(2.6- \\
6.2)\end{array}$ & \\
\hline$\geq 65$ & $\begin{array}{l}12.4 \\
(11.2- \\
13.6)\end{array}$ & & $\begin{array}{l}15.5 \\
(11.4- \\
19.7)\end{array}$ & & $\begin{array}{l}11.4 \\
(9.9- \\
12.8)\end{array}$ & & $\begin{array}{l}13.4 \\
(10.3- \\
16.6)\end{array}$ & & $\begin{array}{l}6.2 \\
(4.4- \\
8.1)\end{array}$ & \\
\hline Sex & & 0.225 & & 0.826 & & 0.027 & & 0.932 & & 0.454 \\
\hline Male & $\begin{array}{l}12.3 \\
(11.1- \\
13.4)\end{array}$ & & $\begin{array}{l}14.9 \\
(12.2- \\
17.6)\end{array}$ & & $\begin{array}{l}10.4 \\
(8.7- \\
12.2)\end{array}$ & & $\begin{array}{l}10.8 \\
(6.1- \\
15.6)\end{array}$ & & $\begin{array}{l}4.9 \\
(3.5- \\
6.3)\end{array}$ & \\
\hline Female & $\begin{array}{l}13.6 \\
(11.7- \\
15.4)\end{array}$ & & $\begin{array}{l}15.5 \\
(12.8- \\
18.3)\end{array}$ & & $\begin{array}{l}12.0 \\
(9.5- \\
14.5)\end{array}$ & & $\begin{array}{l}11.2 \\
(8.5- \\
13.9)\end{array}$ & & $\begin{array}{l}6.2 \\
(3.7- \\
8.8)\end{array}$ & \\
\hline ECOG PS & & 0.013 & & 0.059 & & 0.226 & & 0.574 & & 0.272 \\
\hline 0 or 1 & $\begin{array}{l}13.2 \\
(12.3- \\
14.2)\end{array}$ & & $\begin{array}{l}15.4 \\
(13.1- \\
17.6)\end{array}$ & & $\begin{array}{l}11.6 \\
(10.0- \\
13.2)\end{array}$ & & $\begin{array}{l}11.2 \\
(8.6- \\
13.7)\end{array}$ & & $\begin{array}{l}5.1 \\
(3.5- \\
6.7)\end{array}$ & \\
\hline$\geq 2$ & $\begin{array}{l}9.9 \\
(6.6- \\
13.1)\end{array}$ & & $\begin{array}{l}8.0 \\
(4.6- \\
11.3)\end{array}$ & & $\begin{array}{l}10.2 \\
(8.4- \\
12.0)\end{array}$ & & $\begin{array}{l}13.0 \\
\text { (NA- } \\
\text { NA) }\end{array}$ & & $\begin{array}{l}4.9 \\
(0.3- \\
9.5)\end{array}$ & \\
\hline Stage§ & & 0.003 & & 0.002 & & 0.027 & & 0.971 & & 0.831 \\
\hline
\end{tabular}

Data are presented as months ( $95 \%$ confidence intervals), unless otherwise stated.

Patients in group A received sequential treatment with afatinib and osimertinib, while patients in group $B$ received other therapies following first-line af atinib treatment.

§ Tumor stage was classified based on 8th edition of the American Joint Committee on Cancer staging system.

9 Other tissue types included squamous cell carcinoma in 2 patients, adenosquamous cell carcinoma in 2 patients, and non-small cell lung cancer in 4 patients.

† Patients not presenting with EGFR Del19 and L858R mutations, including de novo T790M mutation, are classified as the "Others" group.

ECOG PS, Eastern Cooperative Oncology Group performance status; EGFR, epidermal growth factor receptor; Del19, deletion 19; meta., metastasis; adj., adjustment, NA, not-available. 


\begin{tabular}{|c|c|c|c|c|c|c|c|c|c|}
\hline \multicolumn{6}{|c|}{ First-line treatment } & \multicolumn{4}{|c|}{ Second-line treatment } \\
\hline 3 and $4 \mathrm{~A}$ & \multicolumn{2}{|l|}{$\begin{array}{l}14.0 \\
(12.2- \\
15.9)\end{array}$} & \multicolumn{2}{|l|}{$\begin{array}{l}17.3 \\
(15.6- \\
19.1)\end{array}$} & \multicolumn{2}{|l|}{$\begin{array}{l}11.6 \\
(9.3- \\
13.9)\end{array}$} & \multicolumn{2}{|l|}{$\begin{array}{l}11.0 \\
(6.5- \\
15.5)\end{array}$} & $\begin{array}{l}5.9 \\
(4.1- \\
7.8)\end{array}$ \\
\hline $4 \mathrm{~B}$ & $\begin{array}{l}12.0 \\
(10.7- \\
13.3)\end{array}$ & & $\begin{array}{l}13.5 \\
(12.0- \\
15.1)\end{array}$ & & $\begin{array}{l}10.4 \\
(8.5- \\
12.4)\end{array}$ & & $\begin{array}{l}11.9 \\
(9.8- \\
13.9)\end{array}$ & & $\begin{array}{l}4.9 \\
(3.5- \\
6.4)\end{array}$ \\
\hline \multicolumn{2}{|l|}{ Smoking } & 0.296 & & 0.155 & & 0.096 & & 0.477 & 0.805 \\
\hline Never & $\begin{array}{l}13.4 \\
(12.3- \\
14.5)\end{array}$ & & $\begin{array}{l}15.5 \\
(13.3- \\
17.8)\end{array}$ & & $\begin{array}{l}11.6 \\
(10.3- \\
12.9)\end{array}$ & & $\begin{array}{l}11.2 \\
(8.6- \\
13.9)\end{array}$ & & $\begin{array}{l}5.9 \\
(3.6- \\
8.2)\end{array}$ \\
\hline Former & $\begin{array}{l}12.2 \\
(10.6- \\
13.7)\end{array}$ & & $\begin{array}{l}12.8 \\
(7.4- \\
18.3)\end{array}$ & & $\begin{array}{l}11.6 \\
(8.7- \\
14.5)\end{array}$ & & $\begin{array}{l}9.4 \\
(5.1- \\
13.7)\end{array}$ & & $\begin{array}{l}4.6 \\
(3.4- \\
5.8)\end{array}$ \\
\hline Current & $\begin{array}{l}13.1 \\
(9.4- \\
16.7)\end{array}$ & & $\begin{array}{l}15.4 \\
(10.4- \\
20.3)\end{array}$ & & $\begin{array}{l}5.5 \\
(2.1- \\
12.7)\end{array}$ & & $\begin{array}{l}\text { NA } \\
\text { (NA- } \\
\text { NA) }\end{array}$ & & $\begin{array}{l}6.3 \\
(3.4- \\
9.2)\end{array}$ \\
\hline \multicolumn{2}{|l|}{ Tissue type } & 0.035 & & 0.214 & & 0.066 & & 0.961 & 0.085 \\
\hline Adenocarcinoma & $\begin{array}{l}12.9 \\
(11.9- \\
13.9)\end{array}$ & & $\begin{array}{l}15.4 \\
(13.2- \\
17.5)\end{array}$ & & $\begin{array}{l}11.4 \\
(10.1- \\
12.6)\end{array}$ & & $\begin{array}{l}11.2 \\
(8.9- \\
13.5)\end{array}$ & & $\begin{array}{l}5.5 \\
(4.0- \\
6.9)\end{array}$ \\
\hline Others 9 & $\begin{array}{l}10.3 \\
(4.6- \\
15.9)\end{array}$ & & $\begin{array}{l}10.7 \\
(10.0- \\
11.4)\end{array}$ & & $\begin{array}{l}3.1 \\
(0.0- \\
8.7)\end{array}$ & & $\begin{array}{l}9.4 \\
(0.6- \\
18.2)\end{array}$ & & $\begin{array}{l}2.1 \\
(1.2- \\
3.1)\end{array}$ \\
\hline \multicolumn{2}{|l|}{ EGFR mutation } & 0.585 & & 0.681 & & 0.657 & & 0.980 & 0.727 \\
\hline Del19 & $\begin{array}{l}13.5 \\
(12.3- \\
14.7)\end{array}$ & & $\begin{array}{l}15.2 \\
(13.1- \\
17.2)\end{array}$ & & $\begin{array}{l}12.4 \\
(10.6- \\
14.1)\end{array}$ & & $\begin{array}{l}11.0 \\
(8.1- \\
13.9)\end{array}$ & & $\begin{array}{l}4.8 \\
(3.3- \\
6.3)\end{array}$ \\
\hline L858R & $\begin{array}{l}13.1 \\
(10.2- \\
16.0)\end{array}$ & & $\begin{array}{l}15.5 \\
(12.7- \\
18.4)\end{array}$ & & $\begin{array}{l}10.0 \\
(6.7- \\
13.4)\end{array}$ & & $\begin{array}{l}11.2 \\
(6.5- \\
16.0)\end{array}$ & & $\begin{array}{l}5.5 \\
(0.6- \\
10.3)\end{array}$ \\
\hline Otherst & $\begin{array}{l}11.4 \\
(9.4- \\
13.4)\end{array}$ & & $\begin{array}{l}12.3 \\
(5.3- \\
19.2)\end{array}$ & & $\begin{array}{l}10.8 \\
(8.5- \\
13.1)\end{array}$ & & $\begin{array}{l}20.0 \\
(1.7- \\
38.4)\end{array}$ & & $\begin{array}{l}6.2 \\
(3.2- \\
9.3)\end{array}$ \\
\hline \multicolumn{10}{|c|}{ Data are presented as months ( $95 \%$ confidence intervals), unless otherwise stated. } \\
\hline \multicolumn{10}{|c|}{$\begin{array}{l}\text { Patients in group A received sequential treatment with afatinib and osimertinib, while patients in group B } \\
\text { received other therapies following first-line afatinib treatment. }\end{array}$} \\
\hline \multicolumn{10}{|c|}{$\S$ Tumor stage was classified based on 8th edition of the American Joint Committee on Cancer staging system. } \\
\hline \multicolumn{10}{|c|}{$\begin{array}{l}\text { Other tissue types included squamous cell carcinoma in } 2 \text { patients, adenosquamous cell carcinoma in } 2 \\
\text { patients, and non-small cell lung cancer in } 4 \text { patients. }\end{array}$} \\
\hline \multicolumn{10}{|c|}{$\begin{array}{l}\text { † Patients not presenting with EGFR Del19 and L858R mutations, including de novo T790M mutation, are } \\
\text { classified as the "Others" group. }\end{array}$} \\
\hline \multicolumn{10}{|c|}{$\begin{array}{l}\text { ECOG PS, Eastern Cooperative Oncology Group performance status; EGFR, epidermal growth factor receptor; } \\
\text { Del19, deletion 19; meta., metastasis; adj., adjustment, NA, not-available. }\end{array}$} \\
\hline
\end{tabular}




\begin{tabular}{|c|c|c|c|c|c|c|c|c|c|c|}
\hline \multicolumn{2}{|c|}{ \# of metastatic organs } & \multirow[t]{2}{*}{0.008} & & \multicolumn{2}{|l|}{0.028} & \multicolumn{2}{|l|}{0.066} & \multicolumn{2}{|l|}{0.048} & \multirow[t]{2}{*}{0.002} \\
\hline $0-1$ & $\begin{array}{l}14.6 \\
(12.7- \\
16.5)\end{array}$ & & $\begin{array}{l}17.2 \\
(15.3- \\
19.2)\end{array}$ & & $\begin{array}{l}12.3 \\
(9.9- \\
14.7)\end{array}$ & & $\begin{array}{l}20.0 \\
(5.7- \\
34.4)\end{array}$ & & $\begin{array}{l}7.0 \\
(2.9- \\
11.1)\end{array}$ & \\
\hline $2-3$ & $\begin{array}{l}12.5 \\
(10.8- \\
14.1)\end{array}$ & & $\begin{array}{l}14.2 \\
(11.7- \\
16.7)\end{array}$ & & $\begin{array}{l}10.4 \\
(8.2- \\
12.6)\end{array}$ & & $\begin{array}{l}13.0 \\
(8.9- \\
17.2)\end{array}$ & & $\begin{array}{l}4.3 \\
(3.1- \\
5.6)\end{array}$ & \\
\hline 4 or more & $\begin{array}{l}10.2 \\
(7.2- \\
13.3)\end{array}$ & & $\begin{array}{l}11.5 \\
(5.7- \\
17.3)\end{array}$ & & $\begin{array}{l}10.2 \\
(7.3- \\
13.2)\end{array}$ & & $\begin{array}{l}8.4 \\
(5.1- \\
11.8)\end{array}$ & & $\begin{array}{l}2.3 \\
(1.4- \\
3.2)\end{array}$ & \\
\hline Adrenal gland meta & & 0.448 & & 0.948 & & 0.039 & & 0.223 & & 0.535 \\
\hline Yes & $\begin{array}{l}10.4 \\
(8.7- \\
12.2)\end{array}$ & & $\begin{array}{l}13.6 \\
(6.1- \\
21.1)\end{array}$ & & $\begin{array}{l}7.1 \\
(0.0- \\
15.6)\end{array}$ & & $\begin{array}{l}10.8 \\
(3.2- \\
18.4)\end{array}$ & & $\begin{array}{l}2.7 \\
(0.0- \\
5.6)\end{array}$ & \\
\hline No & $\begin{array}{l}13.1 \\
(12.1- \\
14.0)\end{array}$ & & $\begin{array}{l}15.2 \\
(13.5- \\
16.8)\end{array}$ & & $\begin{array}{l}11.6 \\
(10.3- \\
13.0)\end{array}$ & & $\begin{array}{l}11.9 \\
(9.1- \\
14.7)\end{array}$ & & $\begin{array}{l}5.5 \\
(4.0- \\
7.0)\end{array}$ & \\
\hline Liver meta. & & 0.002 & & 0.004 & & 0.015 & & 0.003 & & 0.003 \\
\hline
\end{tabular}

Data are presented as months (95\% confidence intervals), unless otherwise stated.

Patients in group A received sequential treatment with afatinib and osimertinib, while patients in group $B$ received other therapies following first-line afatinib treatment.

§ Tumor stage was classified based on 8th edition of the American Joint Committee on Cancer staging system.

9 Other tissue types included squamous cell carcinoma in 2 patients, adenosquamous cell carcinoma in 2 patients, and non-small cell lung cancer in 4 patients.

† Patients not presenting with EGFR Del19 and L858R mutations, including de novo T790M mutation, are classified as the "Others" group.

ECOG PS, Eastern Cooperative Oncology Group performance status; EGFR, epidermal growth factor receptor; Del19, deletion 19; meta., metastasis; adj., adjustment, NA, not-available. 


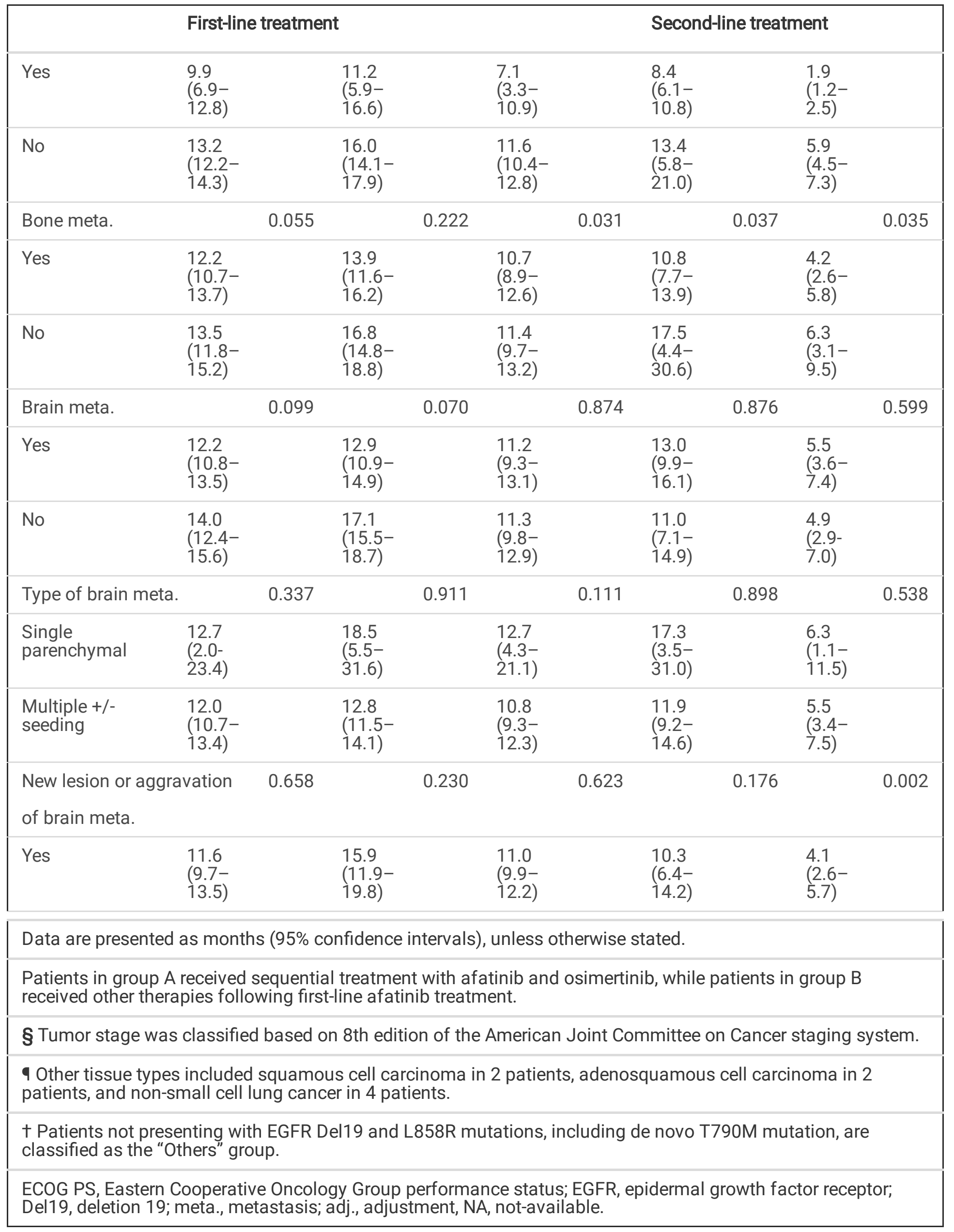




\begin{tabular}{|c|c|c|c|c|c|c|c|c|c|c|}
\hline No & $\begin{array}{l}13.2 \\
(12.2- \\
14.3)\end{array}$ & & $\begin{array}{l}15.1 \\
(12.9- \\
17.4)\end{array}$ & & $\begin{array}{l}11.8 \\
(10.2- \\
13.5)\end{array}$ & & $\begin{array}{l}12.9 \\
(7.8- \\
18.1)\end{array}$ & & $\begin{array}{l}6.6 \\
(3.5- \\
9.4)\end{array}$ & \\
\hline Dose adj. for afatinib & & $\begin{array}{l}< \\
0.001\end{array}$ & & 0.050 & & $\begin{array}{l}< \\
0.001\end{array}$ & & 0.659 & & 0.659 \\
\hline Yes & $\begin{array}{l}13.9 \\
(12.4- \\
15.3)\end{array}$ & & $\begin{array}{l}15.8 \\
(12.8- \\
18.7)\end{array}$ & & $\begin{array}{l}13.0 \\
(11.1- \\
15.0)\end{array}$ & & $\begin{array}{l}11.9 \\
(9.3- \\
14.5)\end{array}$ & & $\begin{array}{l}5.9 \\
(4.1- \\
7.7)\end{array}$ & \\
\hline No & $\begin{array}{l}10.7 \\
(11.7- \\
13.9)\end{array}$ & & $\begin{array}{l}14.6 \\
(12.0- \\
17.3)\end{array}$ & & $\begin{array}{l}9.3 \\
(7.0- \\
11.6)\end{array}$ & & $\begin{array}{l}10.8 \\
(8.0- \\
13.6)\end{array}$ & & $\begin{array}{l}4.6 \\
(3.2- \\
6.0)\end{array}$ & \\
\hline
\end{tabular}

Data are presented as months (95\% confidence intervals), unless otherwise stated.

Patients in group A received sequential treatment with afatinib and osimertinib, while patients in group $B$ received other therapies following first-line afatinib treatment.

§ Tumor stage was classified based on 8th edition of the American Joint Committee on Cancer staging system.

9 Other tissue types included squamous cell carcinoma in 2 patients, adenosquamous cell carcinoma in 2 patients, and non-small cell lung cancer in 4 patients.

† Patients not presenting with EGFR Del19 and L858R mutations, including de novo T790M mutation, are classified as the "Others" group.

ECOG PS, Eastern Cooperative Oncology Group performance status; EGFR, epidermal growth factor receptor; Del19, deletion 19; meta., metastasis; adj., adjustment, NA, not-available.

\subsection{Multivariate Cox PH analysis of factors affecting TOT-1}

In all patients, the multivariate Cox PH model revealed that poor ECOG PS, advanced tumor stage, tissue type other than adenocarcinoma, presence of liver metastasis, and no afatinib dose adjustment were related to decreased TOT1 (Fig. 3). In group A, the hazard ratio (HR) was higher in patients with advanced tumor stage, and the presence of liver metastasis was associated with a marginally significant decrease in TOT-1 (Fig. 4). 


\subsection{Multivariate Cox $\mathrm{PH}$ analysis of factors affecting TOT-2 in group A patients}

In group A patients who received second-line osimertinib treatment, the presence of liver metastasis was associated with a decrease in TOT-2 with an HR of 1.9 (95\% Cl: 1.13-3.3, P = 0.016, Supplementary Fig. 2).

\subsection{ORR-1 and DCR-1}

The results of ORR-1 and DCR-1 are shown in Supplementary Tables 1 and 2, respectively. In the patients who received first-line afatinib treatment, the ORR-1 was 69.9\% (242 of 346) in all patients, 76.3\% (116 of 152) in group A, and $64.9 \%$ (126 of 194) in group B. The DCR-1 in all patients was 93.6\% (324 of 346), with 97.4\% (148 of 152) in group $A$ and $90.7 \%$ (178 of 194) in group B.

\subsection{ORR-2 and DCR-2}

The results of ORR-2 and DCR-2 are shown in Supplementary Tables $\mathbf{3}$ and 4, respectively. The ORR-2 was $38.8 \%$ (50 of 129 patients) in group A and $20.7 \%$ (35 of 169 patients) in group B. DCR-2 was higher than ORR-2 in both groups. The DCR-2 was $93.8 \%$ (121 of 129) in group A and 76.3\% (129/169) in group B.

\subsection{OS, 2-year, and 3-year survival rates}

The estimated OS was 49.1 (95\% Cl: 39.4-58.8) months in all patients with 2-year and 3-year survival rates of $78.1 \%$ and $63.5 \%$, respectively (Supplementary Table 5). The median OS was not reached in group A patients, and it was 38.5 (95\% Cl: 28.8-48.2) months in group B patients (Supplementary Table 6). The OS was significantly longer in group $A$ than in group $B(P=0.0016$, Supplementary Fig. 3).

\section{Discussion}

The present study compared the data of patients who received sequential treatment with afatinib and osimertinib to those of patients who received second-line treatments other than osimertinib. We comprehensively investigated clinical outcomes (i.e., TOT, OS, DCR, and ORR) and robustly evaluated the factors affecting TOT in afatinib and osimertinib treatments. Considering the controversies regarding the use of first-line osimertinib followed by other therapies or first-line first-/second-generation TKIs followed by osimertinib as appropriate options for treating EGFRmutated NSCLC patients ${ }^{15}$, the RWD from RESET might be of interest. RESET included a group of old-age patients with poor performance status and brain metastases, characteristics likely to exclude these patients from RCTs due to strict inclusion criteria. Thus, the results from RESET could be applicable across real-world clinical settings in the management of advanced-stage EGFR-mutated NSCLC patients.

RESET showed that clinical efficacy was better in group A patients than in group B patients. The estimated overall TOT in group A was 33.9 months, which was significantly longer than that in group B (21.3 months). Subgroup analyses also revealed that overall TOT was longer in group A than in group B for all subdivided clinical characteristics, such as smoking status or EGFR mutation type. If we separated first- and second-line treatments, the median TOTs in group A patients were superior to those in group B patients. In addition to TOT, group A was numerically superior to group $B$ with respect to OS, DCR, and ORR for all subdivided clinical characteristics. The AURA-3 clinical trial demonstrated that second-line osimertinib had significantly greater efficacy than pemetrexed plus platinum-based therapy in advanced-stage T790M-mutated NSCLC during treatment with first-line EGFR TKIs such as gefitinib, erlotinib, and afatinib ${ }^{7}$. AURA-3 reported 10.1 months of PFS on osimertinib and 4.4 months of 
PFS on platinum-based pemetrexed therapy, with results comparable to those of RESET, 11.2 months in group A and 5.1 months in group B.

RWD may differ from RCT data for several reasons. RCTs traditionally require strict criteria for study entry, but this would guarantee unbiased distribution of confounding factors, support causality, and provide strong internal validity; thus, evidence from the RCTs has been considered the gold standard. However, because patients with poor performance status, presence of brain metastasis, advanced age, and comorbidities are seldom included, RCTs may suffer from the loss of clinical diversity. Therefore, the importance of RWD in clinical practice has become apparent. RWD can provide supplemental data and additional understanding on top of that from RCTs ${ }^{16}$. In particular, concordance between RWD and RCT data could establish the best approach for managing patients.

However, in terms of sequential therapy with afatinib and osimertinib, only a small number of studies have investigated the clinical characteristics and outcomes in patients with EGFR-mutated NSCLC. In the real-world GioTag study ${ }^{8}$, when using sequential treatment of afatinib and osimertinib, encouraging TOT results were obtained, especially in Asian populations and Del19-positive NSCLC patients. The median TOT was 27.6 months in all patients, 30.3 months in Del19-positive NSCLC patients, and 46.7 months in Asian populations ${ }^{8}$. Updated data on the GioTag study showed that the median OS was 41.3 months and the TTF was 28.1 months ${ }^{17}$. In Del19positive NSCLC patients, the OS was 45.7 months and the TTF was 30.6 months ${ }^{17}$. Although an advantage of the GioTag study is its involvement of a variety of ethnic groups in several countries, it only included 50 Asian patients. During first- and second-line treatments, the overall TOT in all patients within RESET was 26.4 months. The median TOT in group A patients within RESET was estimated to be 33.9 months, which is slightly shorter than that of Asian patients within GioTag, 46.7 months ${ }^{8}$. However, given that only a small number of Asian patients were included in GioTag, the proportion of elderly patients ( $\geq 65$ years) was higher in RESET (43.9\% vs. 34.8\%), and RESET included a higher percentage of patients with baseline brain metastasis (43.9\% vs. $10.3 \%$ ) ${ }^{8}$, the results of RESET are encouraging. The RWD from the RESET study is further supported by another multicenter retrospective study in Japanese patients ${ }^{18}$. In this study, patients sequentially treated with afatinib and osimertinib showed better ORR and DCR than patients treated with other first-generation TKIs (i.e., gefitinib and erlotinib). The above observational studies using RWD highlight the efficacy of sequential treatment with afatinib and osimertinib.

Several RWD trials have estimated the efficacy of afatinib as a first-line treatment option in patients with EGFRmutated NSCLC. The estimated TOT-1 in RESET (15.2 months) is similar to previously reported results from other real-world studies: TOT of 14.0 months for Asian populations in the GioTag study ${ }^{8}$, TTF of 13.1 months in the Japanese population ${ }^{10}$, and TTF of 13.6 months and PFS of 12.4 months in a Taiwanese group ${ }^{13}$. On the contrary, in the Korean population, Kim et al. reported a longer PFS for first-line afatinib treatment (19.1 months) than in our study. This difference might be attributable to the different characteristics of the study subjects. Compared to the same ethnic group in the study by Kim et al., which analyzed 165 Koreans diagnosed with NSCLC, the subjects in our study were older (median age: 61.5 years vs. 57 years) and presented with a slightly higher percentage of brain metastasis $(43.9 \% \text { vs. } 40.6 \%)^{9}$.

RESET assessed several factors affecting TOT-1 using a multivariate Cox PH model. Poor performance status (ECOG PS $\geq 2$ vs. 0-1), advanced tumor stage (AJCC 4B vs. 3), tissue type other than adenocarcinoma, liver metastasis, and no afatinib dose adjustment were shown to be related to decreased TOT-1. Interestingly, afatinib dose adjustment was associated with better outcomes for TOT. It is not clear whether TOT was better due to dose reductions, or whether TOT was worse due to dose maintenance, but a study that investigated the effect of dose 
adjustment on survival outcomes in patients with EGFR-mutated NSCLC reported that patients who received dose reductions experienced higher ORRs ${ }^{19}$. Another RWD study showed that dose adjustment reduced the number and intensity of several side effects, emphasizing that tailored dose modification could help treatment optimization and improve survival outcomes ${ }^{20}$. In addition, a post-hoc analysis of LUX-Lung 3 and 6 trials showed that dose reduction was more common in female, old-age, low-weight, and Asian-Japanese patients ${ }^{21}$. Dose reduction led to fewer adverse events and less treatment discontinuation.

Notably, the TOT-1 in group A patients was greater than that in group B patients. Tanaka et al. reported that PFS was significantly longer in patients who developed the T790M mutation after the onset of first-line afatinib therapy than in those who did not develop the mutation ${ }^{22}$. Indeed, in RESET, the percentage of T790M development after first-line afatinib was greater in group A than in group B patients: 126/131 (96.2\%) in group A and 8/81 (9.9\%) in group B. This result is consistent with another study showing that OS was significantly longer in patients with the T790M mutation than in those without it ${ }^{23}$. Although the mechanism of resistance to EGFR-TKIs might be heterogeneous, the slow growth rate of T790M-harboring cells could partially explain this observation ${ }^{24}$. These findings might account for the better survival outcomes in patients who developed the T790M mutation after first-line afatinib treatment, indicating potential association between a longer treatment period and development of the mutation.

Several EGFR-TKIs have been developed to address the problem of EGFR mutations following therapy. Currently, the standard treatment option for patients with EGFR-mutated advanced-stage NSCLC is an EGFR-TKI. Considering its superior efficacy in terms of PFS, OS, central nervous system activity, and adverse events, clinicians prefer osimertinib as first-line therapy ${ }^{25}$. Despite these benefits, inevitably acquired mutations during osimertinib therapy, such as the C797S mutation ${ }^{26}$, as well as the interpatient, intratumoral, and intertumoral heterogeneity of NSCLC ${ }^{27}$, complicate the optimal therapeutic determination of EGFR-TKI sequence. The optimal sequence of treatment remains controversial ${ }^{15}$. In particular, in the National Health Insurance of South Korea, osimertinib is only approved for second-line treatment after failure of other first-line EGFR-TKI treatments. Therefore, sequential treatment of firstor second-generation EGFR-TKIs with the third-generation EGFR-TKI, osimertinib, is a possible alternative.

The RESET study has limitations. First, the main limitation resulted from RESET's retrospective nature. Selection bias or misclassification existed. To mitigate this problem, subgroup analyses were performed to identify any potential factors significantly affecting survival outcomes. The results of multicenter hospital-based surveillance from RESET could provide insight into the universality of the efficacy of sequential treatment with afatinib and osimertinib. Second, TOT-2 was shorter than TOT-1, which is opposite to the finding in GioTag. TOT-1 in GioTag might have been rather short due to inclusion criteria and drug availability. And also, this may have originated from the short observation period of osimertinib treatment in RESET, in which survival data were not matured at the time of analysis; further data collection and analysis may be warranted. Third, five of the group A patients did not develop the T790M mutation and received osimertinib treatment, while seven of the group B patients developed T790M and did not receive the treatment. Fourth, because 377 patients were excluded, it was not feasible to obtain more detailed information, such as the percentage of patients who received second-line treatment or the frequency of T790M development. In the future, we are planning to also collect data in group A and B patients and in excluded patients who received first-line afatinib treatment. Despite these limitations, to the best of our knowledge, RESET is the first multicenter study in South Korea based on real-world experience, and its applicability to real clinical practice, especially for Asian populations, could allow better patient management and improved survival outcomes in patients with EGFR-mutated advanced NSCLC. 


\section{Conclusions}

Real-world Experience of SEquential Treatment of afatinib and osimertinib (RESET) is the first multicenter study in South Korea in patients with EGFR-mutated advanced NSCLC, comprehensively comparing the RWD of sequential treatment with afatinib and osimertinib to the RWD of other second-line treatments. Osimertinib after first-line afatinib treatment was superior to other regimens as second-line treatments in terms of TOT, OS, ORR, and DCR. Our results show the feasibility of sequential treatment with afatinib and osimertinib in patients with EGFR-mutated advanced NSCLC, maximizing sustained clinical benefit and minimizing exposure to chemotherapy.

\section{Materials And Methods}

\subsection{Patients, design, and data collection}

Real-world Experience of SEquential Treatment of afatinib and osimertinib (RESET) is a retrospective multicenter observational study in South Korea across 16 medical centers. Electronic medical records from October 2014 to October 2019 of patients who met the following inclusion criteria were reviewed: (囚) age $\geq 19$ years with EGFRmutated TKI-naïve advanced-stage NSCLC that was newly diagnosed pathologically and (ii) treated first-line with afatinib and second-line with either osimertinib or other treatments. The NSCLC advanced stages were defined as stages 3B, 3C, 4A, and 4B, which are not eligible for standard operative procedures, based on the 8th edition of the American Joint Committee on Cancer (AJCC) staging system. Patients who were not treated with afatinib as firstline therapy or osimertinib as second-line therapy were excluded. Patients who were initially treated with chemoradiotherapy were also excluded.

Baseline demographic characteristics (age, sex, and Eastern Cooperative Oncology Group performance status [ECOG PS]) were collected. Smoking status was categorized into never, former, and current smokers according to the classification of the National Health Interview Survey. The date of diagnosis, initiation of first-line afatinib, information on EGFR mutation (i.e., presence/absence and profile), number of metastatic organs, existence of specific organ metastasis, and dose modification of afatinib were recorded. For EGFR mutation analysis, the peptic nucleic acid-mediated real-time polymerase chain reaction (PCR) clamping method (Panagene, Daejeon, Korea) or the Roche Cobas EGFR mutation test (Roche Molecular Systems, Pleasanton, CA, USA) were used. Follow-up data regarding the date and regimen of second-line treatment and new lesions or aggravation of brain metastasis were also collected.

\subsection{Ethical approval}

The study and protocol were approved by the Institutional Review Board (IRB) of the Kosin University Gospel Hospital (KUGH no. 2019-07-038). The study was conducted following the Declaration of Helsinki. All procedures were performed in accordance with the relevant guidelines and regulations. Informed consent was waived due to the retrospective nature of this study, and the approval was gained by the above mentioned IRB.

\subsection{Outcomes and measurements}

The primary outcome was time on treatment (TOT). TOT-1 was defined as the time from the first dose of afatinib to tumor progression, TOT-2 was defined as the time from the first dose of second-line therapy to tumor progression or 
death during the treatment, and overall TOT was defined as the length between the first dose of afatinib and tumor progression or death during the second-line treatment.

The secondary outcomes were as follows: ( $(\mathbb{)})$ ORR-1, defined as the ratio of total patients who received afatinib to patients experiencing complete remission (CR) or partial remission (PR) after the first evaluation of tumor response, which was defined based on Response Evaluation Criteria in Solid Tumors (RECIST) version $1.1^{14}$, (ii) ORR-2 for second-line treatment, (iii) disease control rate-1 (DCR-1), defined as the ratio of total patients receiving afatinib to patients with CR, PR, and stable disease (SD) after the first evaluation of tumor response defined based on RECIST

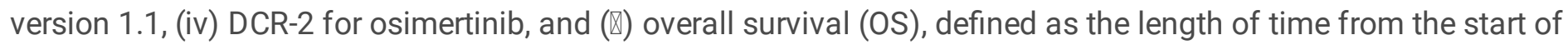
afatinib to death from any cause. Patients still on treatment were censored at the time of data collection.

\subsection{Statistical analysis}

The baseline patient characteristics were descriptive. Chi-squared and Fisher's exact tests were used to compare the differences between categorical variables. TOT and OS were estimated with the Kaplan-Meier method and differences in time distributions were compared using the log-rank test; the estimated median time (months) and 95\% confidence interval $(\mathrm{Cl})$ are presented. The Cox proportional hazards $(\mathrm{PH})$ model was used to investigate the effect of independent variables on survival outcome. Variables with $\mathrm{P}<0.05$ in the univariate Cox $\mathrm{PH}$ model were included in the multivariate Cox PH model. All statistical analyses were performed using IBM SPSS Statistics for Windows, version 25.0 (IBM Corp., Armonk, New York), and R software version 4.0.3 for Windows (R Development Core Team). P-value cutoffs were trichotomized into $<0.01,<0.05$, and $<0.10$, considering them as highly significant, significant, and marginally significant.

\section{Declarations}

\section{Acknowledgements}

We would like to thank Editage (www.editage.co.kr) for English language editing.

\section{Authorship contribution}

T Kim: Writing - original draft, Methodology, Software, Formal analysis, Data Curation, Visualization. TW Jang: Conceptualization, Funding acquisition, Writing - review \& editing, Supervision, Project administration. All authors: Investigation, Validation. All authors discussed the results and approved the final version of the manuscript.

\section{Disclosure:}

All authors declare that they have no conflicts of interest.

\section{Funding}

This study was funded by Boehringer Ingelheim Korea. The company had no role in the data collection, data analysis, preparation of the manuscript, or decision to publish. 


\section{References}

1. Jung, K. W., Won, Y. J., Kong, H. J. \& Lee, E. S. Prediction of Cancer Incidence and Mortality in Korea, 2018. Cancer Res. Treat.50, 317-323 https://doi.org/10.4143/crt.2018.142 (2018).

2. Bray, F. et al. Global cancer statistics 2018: GLOBOCAN estimates of incidence and mortality worldwide for 36 cancers in 185 countries. CA Cancer J. Clin.68, 394-424 https://doi.org/10.3322/caac.21492 (2018).

3. Sequist, L. V. et al. Phase III study of afatinib or cisplatin plus pemetrexed in patients with metastatic lung adenocarcinoma with EGFR mutations. J. Clin. Oncol.31, 3327-3334 https://doi.org/10.1200/jco.2012.44.2806 (2013).

4. Park, K. et al. Afatinib versus gefitinib as first-line treatment of patients with EGFR mutation-positive non-smallcell lung cancer (LUX-Lung 7): a phase 2B, open-label, randomised controlled trial. Lancet Oncol.17, 577-589 https://doi.org/10.1016/s1470-2045(16)30033-x (2016).

5. Arcila, M. E. et al. Rebiopsy of lung cancer patients with acquired resistance to EGFR inhibitors and enhanced detection of the T790M mutation using a locked nucleic acid-based assay. Clin. Cancer Res.17, 1169-1180 https://doi.org/10.1158/1078-0432.Ccr-10-2277 (2011).

6. Yang, J. C. et al. Osimertinib in Pretreated T790M-Positive Advanced Non-Small-Cell Lung Cancer: AURA Study Phase II Extension Component. J. Clin. Oncol.35, 1288-1296 https://doi.org/10.1200/jco.2016.70.3223 (2017).

7. Mok, T. S. et al. Osimertinib or Platinum-Pemetrexed in EGFR T790M-Positive Lung Cancer. N. Engl. J. Med.376, 629-640 https://doi.org/10.1056/NEJMoa1612674 (2017).

8. Hochmair, M. J. et al. Sequential treatment with afatinib and osimertinib in patients with EGFR mutationpositive non-small-cell lung cancer: an observational study. Future Oncol.14, 2861-2874 https://doi.org/10.2217/fon-2018-0711 (2018).

9. Kim, Y. et al. Efficacy and Safety of Afatinib for EGFR-mutant Non-small Cell Lung Cancer, Compared with Gefitinib or Erlotinib. Cancer Res. Treat.51, 502-509 https://doi.org/10.4143/crt.2018.117 (2019).

10. Fujiwara, A. et al. A Retrospective Comparison of the Clinical Efficacy of Gefitinib, Erlotinib, and Afatinib in Japanese Patients With Non-Small Cell Lung Cancer. Oncol. Res.26, 1031-1036

https://doi.org/10.3727/096504018x15151523767752 (2018).

11. Deng, W. et al. Comparing overall survival between first generation EGFR-TKIs and chemotherapy in lung cancer patients with Del19/L858R. Chinese journal of cancer research = Chung-kuo yen cheng yen chiu.28, 339-347 https://doi.org/10.21147/j.issn.1000-9604.2016.03.08 (2016).

12. Kuan, F. C. et al. Analysis of progression-free survival of first-line tyrosine kinase inhibitors in patients with nonsmall cell lung cancer harboring leu858Arg or exon 19 deletions. Oncotarget.8, 1343-1353 https://doi.org/10.18632/oncotarget.13815 (2017).

13. Lin, Y. T. et al. Clinical outcomes and secondary epidermal growth factor receptor (EGFR) T790M mutation among first-line gefitinib, erlotinib and afatinib-treated non-small cell lung cancer patients with activating EGFR mutations. Int. J. Cancer.144, 2887-2896 (2019).

14. Schwartz, L. H. et al. RECIST 1.1-Update and clarification: From the RECIST committee. European journal of cancer (Oxford, England: 1990) 62, 132-137, doi:10.1016/j.ejca.2016.03.081 (2016).

15. Recondo, G., Facchinetti, F., Olaussen, K. A., Besse, B. \& Friboulet, L. Making the first move in EGFR-driven or ALK-driven NSCLC: first-generation or next-generation TKI? Nat. Rev. Clin. Oncol.15, 694-708 https://doi.org/10.1038/s41571-018-0081-4 (2018). 
16. Cheema, P. K. \& Kuruvilla, S. Fulfilling the potential of real-world evidence for lung cancer in Canada. Curr. Oncol.26, 157-159 https://doi.org/10.3747/co.26.5151 (2019).

17. Hochmair, M. J. et al. Sequential afatinib and osimertinib in patients with EGFR mutation-positive non-small-cell lung cancer: updated analysis of the observational GioTag study. Future Oncol.15, 2905-2914 https://doi.org/10.2217/fon-2019-0346 (2019).

18. Tamiya, M. et al. Which Is Better EGFR-TKI Followed by Osimertinib: Afatinib or Gefitinib/Erlotinib? Anticancer Res.39, 3923-3929 https://doi.org/10.21873/anticanres.13544 (2019).

19. Ho, G. F. et al. Real-world experience of first-line afatinib in patients with EGFR-mutant advanced NSCLC: a multicenter observational study. BMC Cancer.19, 896 https://doi.org/10.1186/s12885-019-6107-1 (2019).

20. Halmos, B. et al. Impact of afatinib dose modification on safety and effectiveness in patients with EGFR mutation-positive advanced NSCLC: Results from a global real-world study (RealGiDo). Lung Cancer.127, 103111 https://doi.org/10.1016/j.lungcan.2018.10.028 (2019).

21. Yang, J. C. et al. Effect of dose adjustment on the safety and efficacy of afatinib for EGFR mutation-positive lung adenocarcinoma: post hoc analyses of the randomized LUX-Lung 3 and 6 trials. Ann. Oncol.27, 21032110 https://doi.org/10.1093/annonc/mdw322 (2016).

22. Tanaka, K. et al. Acquisition of the T790M resistance mutation during afatinib treatment in EGFR tyrosine kinase inhibitor-naïve patients with non-small cell lung cancer harboring EGFR mutations. Oncotarget.8, 68123-68130 https://doi.org/10.18632/oncotarget.19243 (2017).

23. Ke, E. E. et al. A Higher Proportion of the EGFR T790M Mutation May Contribute to the Better Survival of Patients with Exon 19 Deletions Compared with Those with L858R. J. Thorac. Oncol.12, 1368-1375 https://doi.org/10.1016/j.jtho.2017.05.018 (2017).

24. Chmielecki, J. et al. Optimization of dosing for EGFR-mutant non-small cell lung cancer with evolutionary cancer modeling. Sci. Transl. Med.3, 9059 https://doi.org/10.1126/scitranslmed.3002356 (2011).

25. Soria, J. C. et al. Osimertinib in Untreated EGFR-Mutated Advanced Non-Small-Cell Lung Cancer. N. Engl. J. Med.378, 113-125 https://doi.org/10.1056/NEJMoa1713137 (2018).

26. Ricordel, C., Friboulet, L., Facchinetti, F. \& Soria, J. C. Molecular mechanisms of acquired resistance to thirdgeneration EGFR-TKIs in EGFR T790M-mutant lung cancer. Ann. Oncol.30, 858 https://doi.org/10.1093/annonc/mdy222 (2019).

27. Zito Marino, F. et al. Molecular heterogeneity in lung cancer: from mechanisms of origin to clinical implications. Int. J. Med. Sci.16, 981-989 https://doi.org/10.7150/ijms.34739 (2019).

\section{Figures}




\section{Overall TOT}

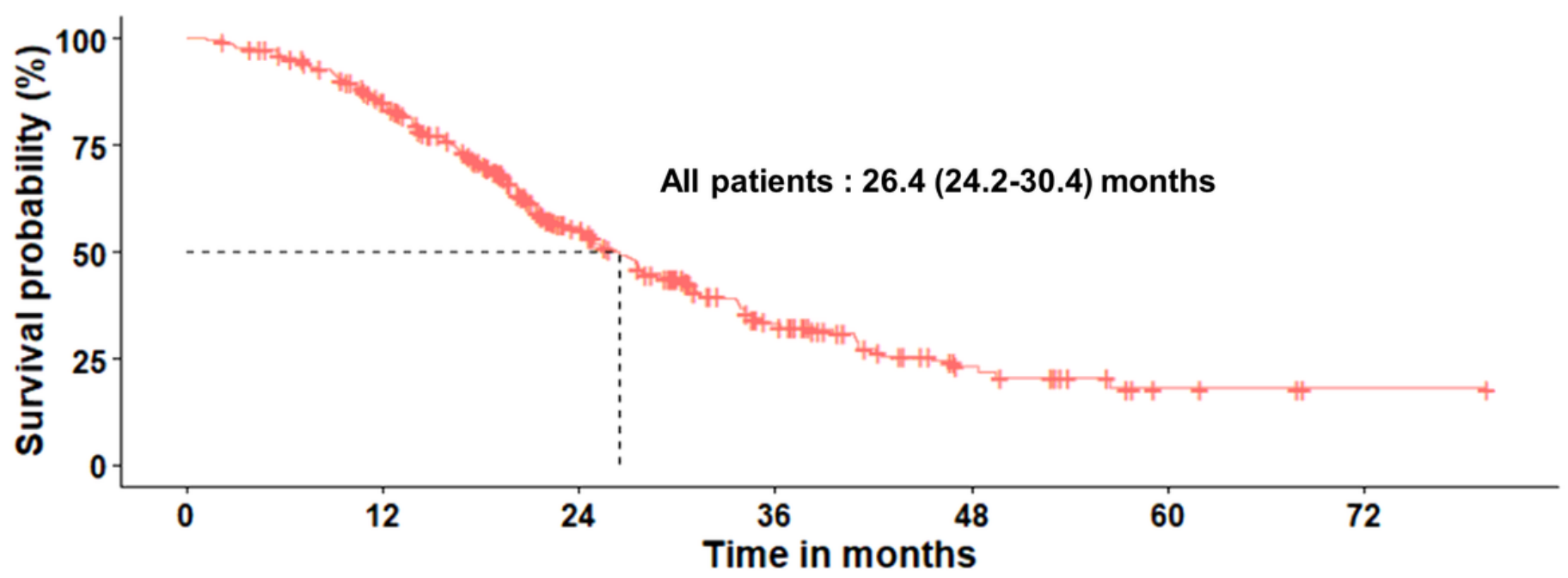

\section{Overall TOT}

$$
\text { Strata + Group A + Group B }
$$

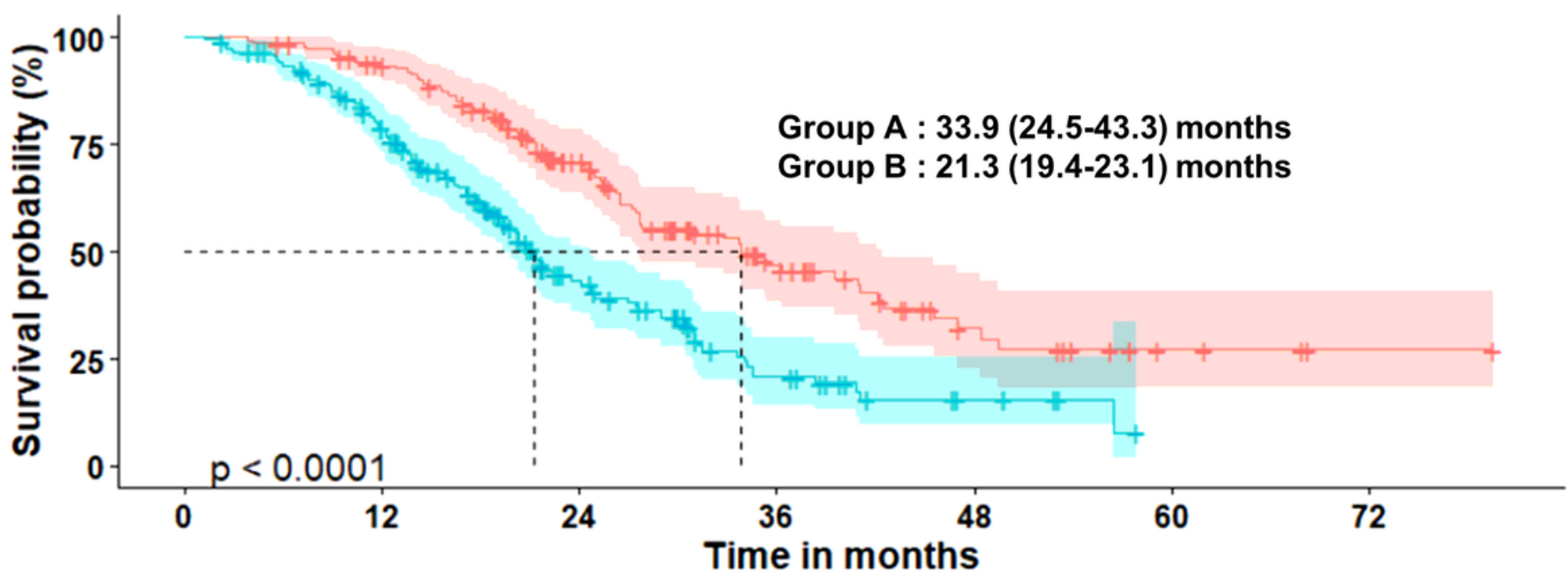

Figure 1

Top) Overall time-on-treatment (TOT) for first- and second-line treatment in all patients $(\mathrm{n}=360)$ with advanced NSCLC harboring EGFR mutations. Bottom) Overall TOT, using osimertinib (group A, $n=154$ ) and other medications (group $B, n=206$ ) as second-line treatment. 


\section{TOT on afatinib}

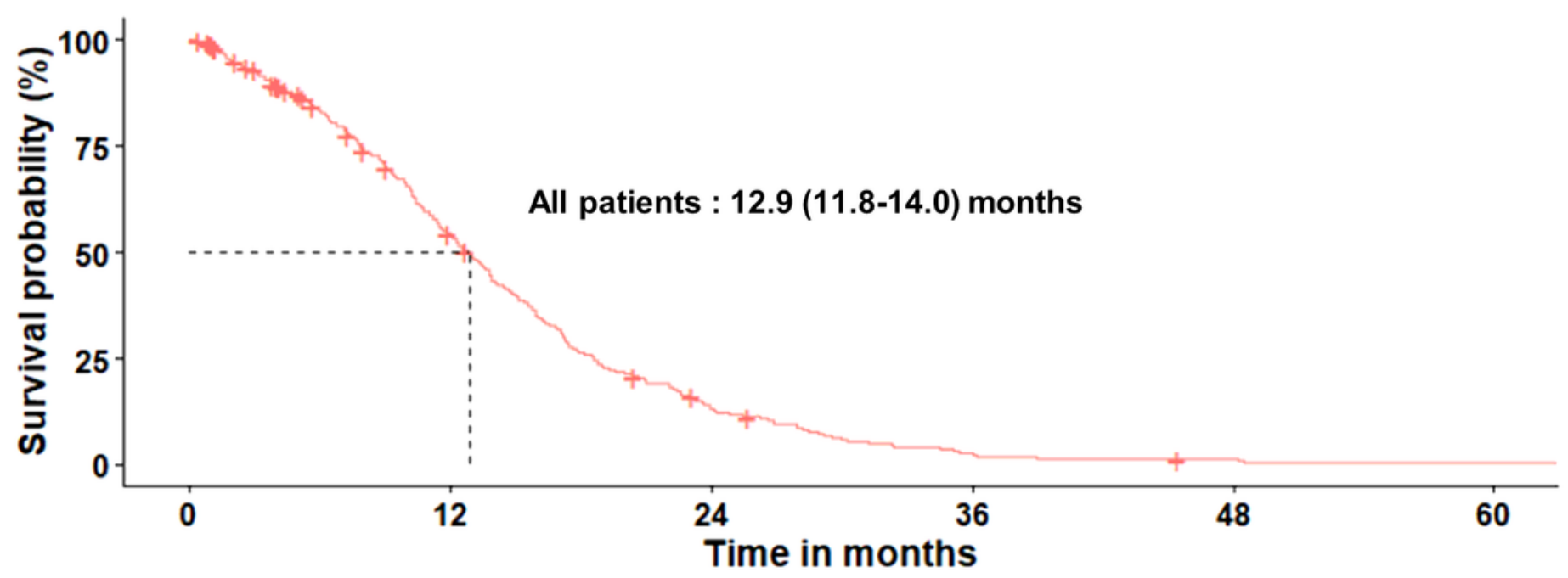

\section{TOT on afatinib}

$$
\text { Strata + Group A + Group B }
$$

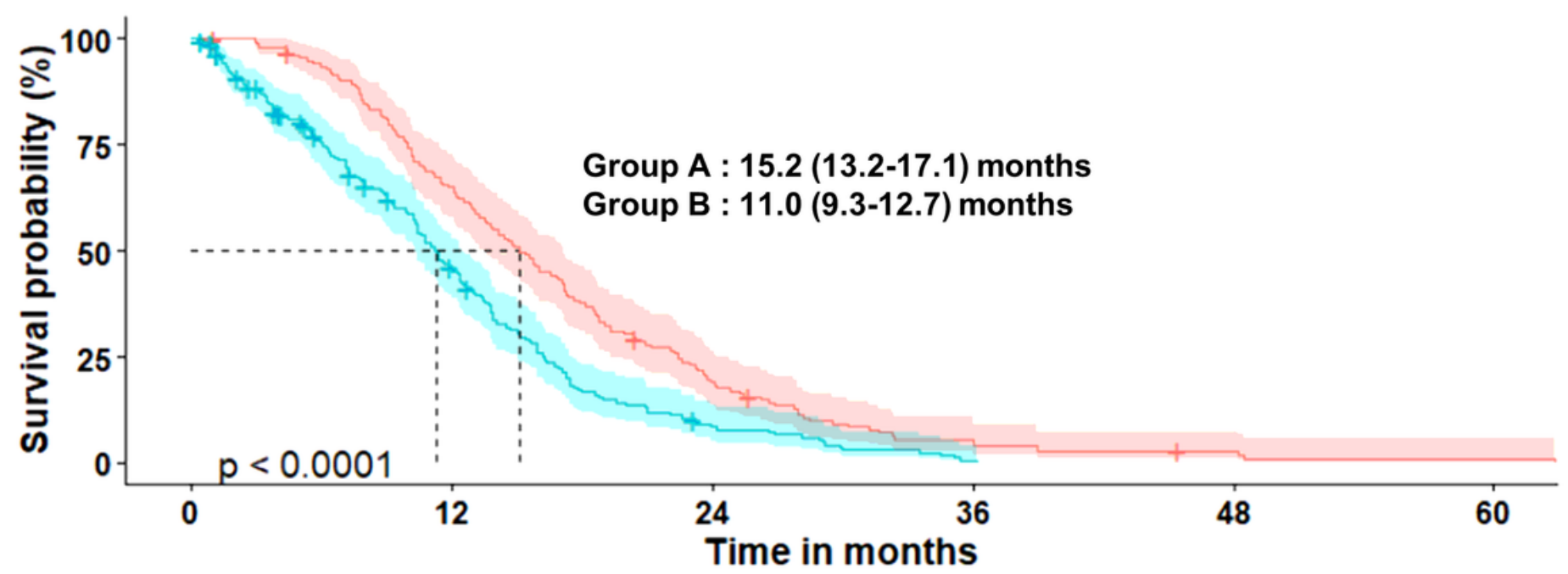

Figure 2

Top) Time-on-treatment (TOT) for first-line treatment with afatinib in all patients $(\mathrm{n}=360)$ with advanced NSCLC harboring EGFR mutations. Bottom) TOT with afatinib, when osimertinib (group $A, n=154$ ) and other medications (group $B, n=206$ ) are used as second-line treatment. 


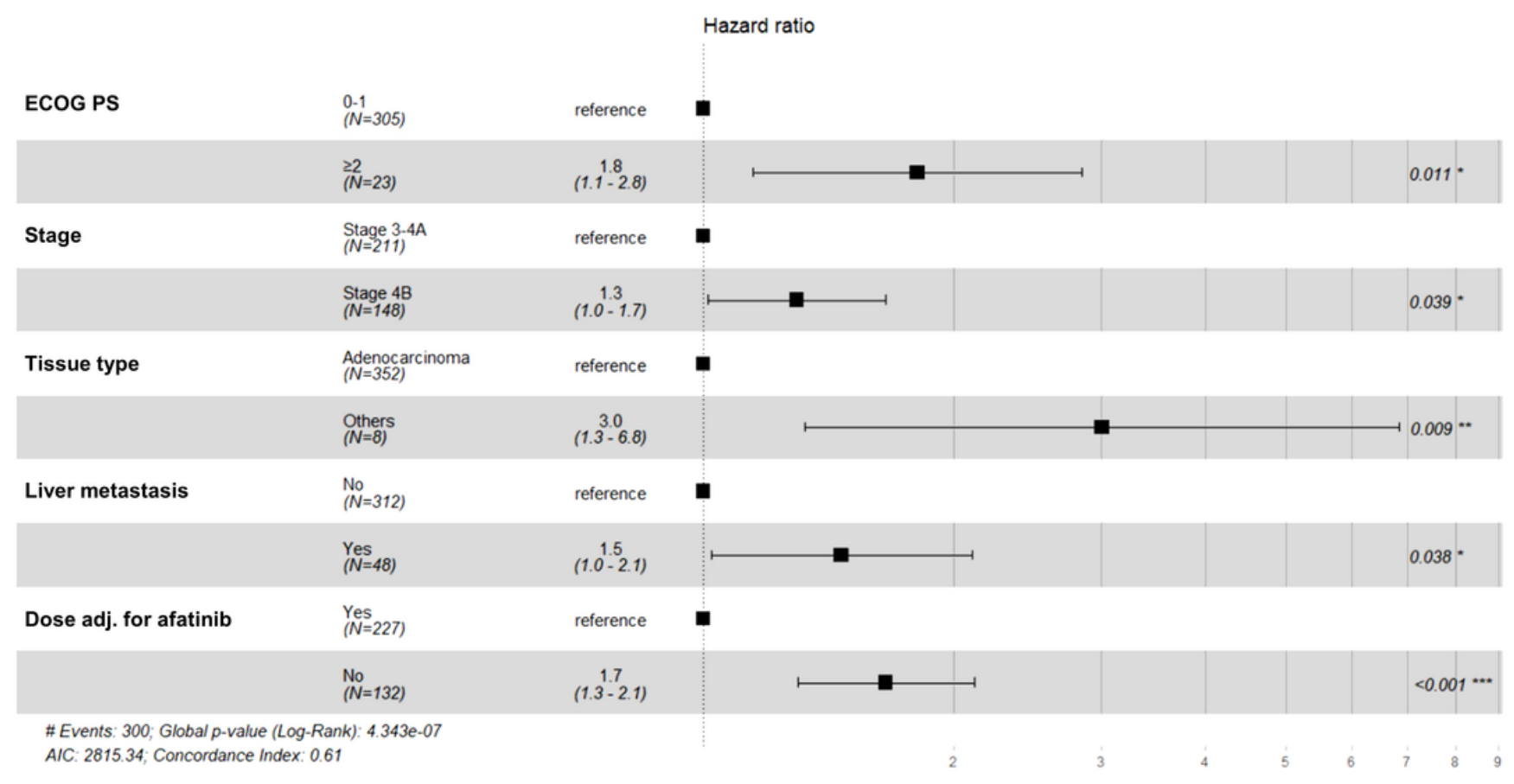

\section{Figure 3}

Multivariate Cox proportional hazards regression analysis of factors affecting time on afatinib treatment in all patients $(n=360)$ with advanced NSCLC harboring EGFR mutations.

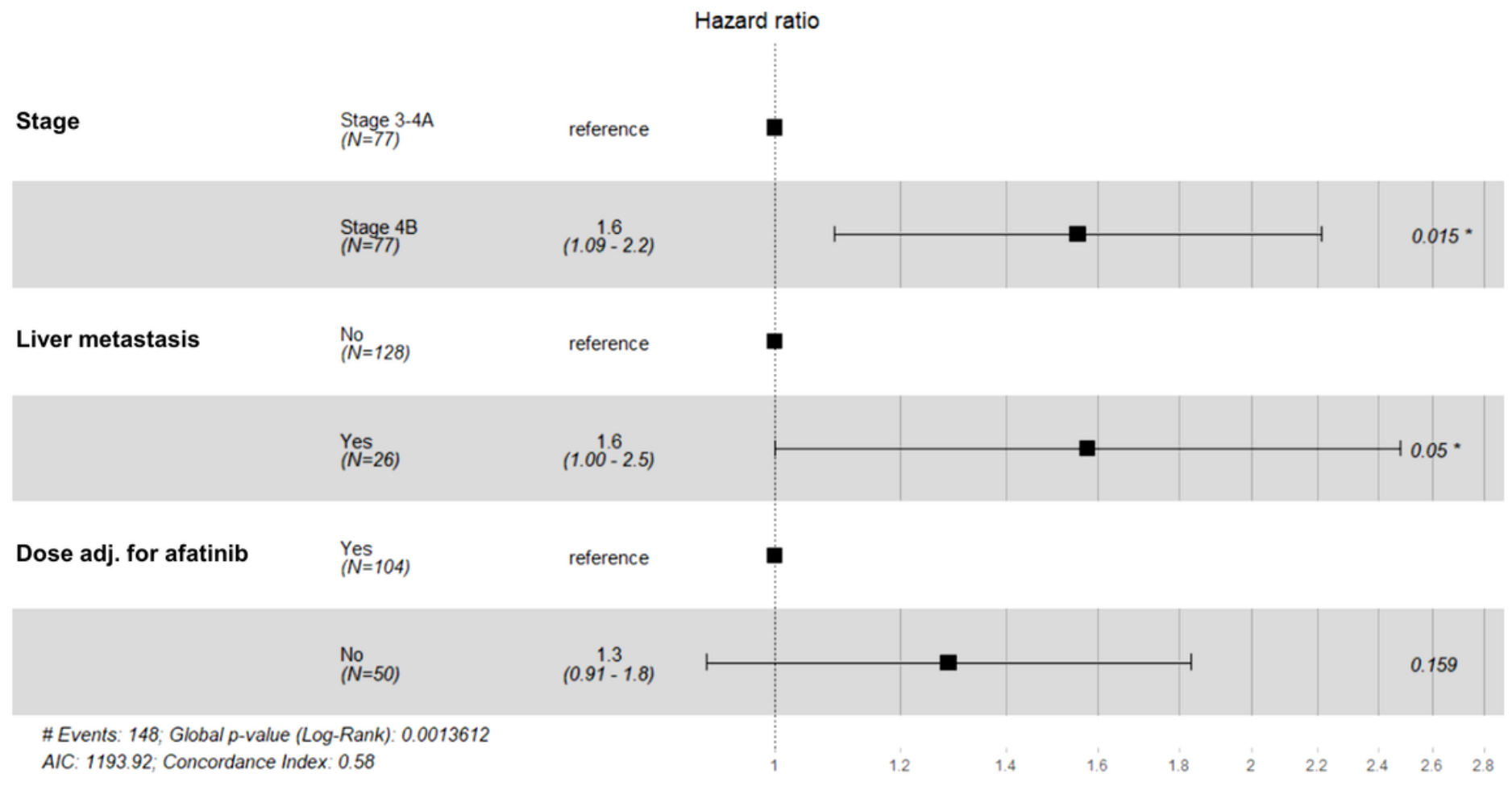

Figure 4 
Multivariate Cox proportional hazards regression analysis of factors affecting the time on first-line afatinib treatment, in patients with advanced NSCLC, harboring EGFR mutations, and receiving osimertinib as a second-line treatment $(n=206)$.

\section{Supplementary Files}

This is a list of supplementary files associated with this preprint. Click to download.

- SupplementaryFigure1.tif

- SupplementaryFigure2.tif

- SupplementaryFigure3.tif

- SupplementaryInformationandTables.docx 